the two point test to be made (Fig. 4). The distance between the two points may be read on the millimeter scale. A camel's hair brush, $C$, to test tactile sensation is available by unscrewing and reversing the screw, 3 , as shown in Figures 2 and 4 . The threads (4) in Figure 2 are made to hold the head of the hammer, $A$, when this part is unscrewed when testing pain sensation or the two point test. Figure 3 shows the hammer in place. It was thought that by having a definite place to put this part when not in use there would be less likelihood of losing it and less need to hunt for it among the bedclothes or in one's pockets. The point $D$ in Figure 2 will be found sufficiently rough and sharp to usc in testing for the abdominal, cremasteric and Babinski and allied reflexes. The rubber tip and the camel's hair brush are parts which wear, and these are therefore made so as to be replaceable. The hammer is made by the Klett Manufacturing Company, 202 East Forty-Sixth Street, New York City.

515 Park Avenue.

\section{RECTAL BINDER}

\section{A. J. Chisholm, M.D., Denver}

Having had difficulty in finding a suitable binder for use in cases following rectal operations, especially fistula, when the patient is required to wear a gauze pad for a few weeks, I designed the binder shown in the accompanying illustration, which, I believe, is superior to any so far used. The cost is nomirral.

The binder, which is adjustable, is supported by an arrangement of tapes fastened to a waistband. In detail, it is a cloth band $(a)$ permanently $f$ astened by one end, the back, to an elastic belt $(b)$, which is provided with a buckle for "waist" adjustment. The other end of the cloth band is hemmed $(c)$, so that this end of the band can slide on an elastic tape (d). The elastic tape is made in two parts having a buckle connection for adjustment, and its extreme ends slide on

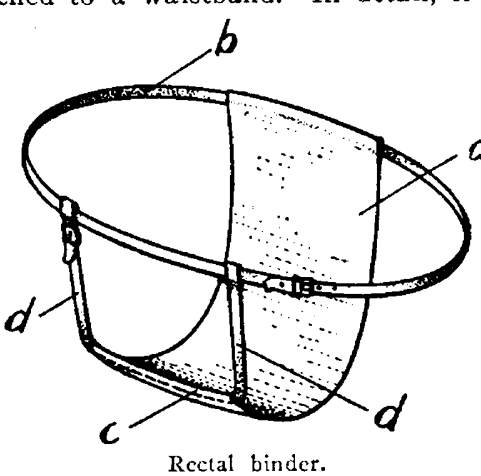
the elastic waistband $(b)$. The plain end of the elastic tape $(d)$ is passed through the hem of the cloth band $(a)$, and is then adjusted to the right tension by means of the buckle on the end of the other part of this tape.

The features of this binder are thought to be new and useful in that the binder retains rectal dressings and at the same time provides ample adjustment for the convenience of the patient. Binders can be obtained from the Butterfield Surgical Company, Denver.

830 Metropolitan Building.

\section{GUMMA OF THE FOREHEAD WITH RINGWORM: REPORT OF A CASE}

Herman Goodman, M.D., New York

The recent report of penile chancre with ringworm ${ }^{1}$ recalls the following case:

M. S., aged 32, married, presented himself at the New York Skin and Cancer Hospital (in the service of Dr. C. M. Williams, absent on military duty), June 11, 1917, with an cruption on the forehead, over the right eyebrow but not involving the hair there. The lesion was about the area of a half dollar, raised and rather firm, without evidence of vesiculation at the border or of ulceration in any part. The patient said that, except for its appearance, the lesion was

1. Eichenlaub, F. J.: Penile Chancre with Ringworm, J. A. M. A. 79: 1518 (Oct. 28) 1922 not the source of any subjective symptoms, such as itching or burning. The lesion had reached the size mentioned about two wecks previously. No history of syphilis was elicited. The wife and only child were supposedly healthy.

My clinical diagnosis was gumma; but, to satisfy one of the associates that there was no evidence of ringworm, scrapings were examined. To my surprise, on microscopic examination I found spores, and the culture on Sabouraud's medium was positive. The growth presented the morphologic characteristics of small spore ringworm, but no further differentiation was made. This culture was carried on for a period, and it was utilized in some experiments to determine the lethal qualities of drugs commonly employed in the treatment of ringworm of the glabrous skin, as well as in the repetition of the experiment to show that the roentgen ray does not deter the growth (the latter performed with Dr. W. J. Young).

The Wassermann reaction was reported three plus on blood withdrawn on the day of admission and a month later. A test of the wife's blood was also reported strongly positive. The patient received antisyphilitic treatment (arsphenamin), and the lesion entirely cleared. I examined the child, bit could find no tinea. How the ringworm infection was acquired was not determined. The patient was a chauffeur; and there were no pets at home. It seems that the primary lesion of the skin, the gumma, allowed any chance contamination a better field for growth than the unafected skin. In this manner, other skin affections may prove to have tinea or yeast thriving on the surface, or even deeper in the lesion; yet these organisms are not causative or primary in nature, but purely secondary and contaminating associations. Indeed, scrapings from apparently normal skin have been known to give growth on culture, either for the dermatophytes or the yeasts.

15 Central Park West.

\section{EXCESSIVE GOLF FOLLOWED BY MUSCULOSPIRAL PALSY \\ Cornelius C. Wholey, M.D., Pittsaugh}

A man, aged 35 , consuited me because of severe pain which would come on as soon as he lay down, and which disturbed his sleep. The pain was located to the left of the spine over the lower part of the neck. The peculiarity of the pain was that it would largely disappear when the patient was up and about his work, but, no matter what position he lay in, it would continue. Examination revealed a pronounced weakness involving all of the extensor muscles of the left upper extremity. The ability to extend the wrist against resistance was very weak. In fact, the wrist was held in a partially wrist-drop position. Extension of the fingers, and at the clbow, and at the shoulder were proportionately weak. The hand grip was also very much reduced in strength. There was a similar weakness in the power of supinating the forearm. Testing for sensation revealed that there was numbness over the radial surface of the thumb, and the patient complained of having noticed prickling and numbness in the tip of this and the index finger. The patient had noticed an area, of about 4 inches in length and 2 inches in width, of numbness along the middle dorsal surface of the forearm, which I was able to demonstrate by testing for touch and pain.

It was evident that there was an involvement of the entire musculature supplied by the musculospiral nerve. All of the deep reflexes of the extremity were exaggerated. There was some tenderness on pressure over the nerve trunks. There was a small area of marked tenderness on pressure between the inner, upper angle of the scapula and the lower cervical spine. The sharp pain of which the patient complained and which became so pronounced on lying down was mainly over this area.

The peculiar circumstance of the pain being so noticeably aggravated by the patient's lying down, together with the existence of the tender area along the lower neck behind, made me regard the nerve involvement as being largely of 
root origin. It seemed likely that a slight pull on the nerve roots was in some way brought about by the change of position, this accounting for the severe pain when the patient was recumbent.

This man gave a history of leading a very active life, and of being an enthusiastic golf player. He had occasionally - noticed slight symptoms of pain in the left thumb and shoulder, and slight weakness in the left arm as far back as a month, but for the two weeks preceding his visit the symptoms had been severe. I learned on further inquiry that all his life he had been unusually active, and, regardless of this, he rarely experienced any sense of fatigue. Roentgen-ray examination revealed no pathologic changes in the cervical spine covering the origin of the brachial plexus, and urinary and blood tests were negative for syphilitic and kidney involvement.

The paralysis in this case can be explained in this way: The patient was constitutionally of the hyperactive type. Such persons are often in a more or less exhausted nervous condition without being aware of the fact. This patient was in such a state of fatigue when his palsy was precipitated. He had been crowding in his daily golf, in spite of excessively long and arduous business hours. And there was superadded to his general nerve fatigue a focalized nerve strain sufficient to induce a neuritis.

When one considers the grip of the golfer on his club, together with the nature of the swing given the club, it becomes evident that it is the musculature supplied by the musculospiral nerve that is very noticeably called into action, namely, that of extension of the upper extremity throughout its entire length. When this man finally took a general rest, the symptoms disappeared after several weeks, and he was able to resume golf without any difficulty. The numbness over the thumb and forearm remained to some extent two months after the onset of the trouble.

The musculospiral is one of the most frequently involved of all the nerves of the extremities. Fortunately, however, in paralysis of this nerve the outlook is most favorable. In the majority of cases, paralysis occurs in persons from toxic causes; and even when there has been a specific strain on the nerve, as in our case, one often finds a history of alcohol, exhaustion or some chronic toxic condition. Sudden violent contraction of the extensor muscles sometimes precipitates this palsy, and in the light of this fact, it is worthy of note that in the "follow-through" swing of the golfer's club we have conditions somewhat comparable; "to which is added the repeated full stretch and tug on the nerve trunks, and the latter possibly on the roots of the brachial plexus as well.

818 Westinghouse Building.

\section{FETAL TERATOMA IN A CHILD}

\section{William H. German, M.D., Chicago}

D. S., a girl, aged 6 years and 10 months, gave no history of illness, except that she had scarlet fever in 1921. She is said to have complained of occasional pain in the abdomen, but it was not thought serious, and she appeared normal.

July 9, 1922, she accidentally fell over a small rocking chair, and soon developed severe pain in the umbilical and left abdominal regions. July 30 , examination revealed general abdominal rigidity and tension, tenderness over the appendix and a distinct tumor in the left abdomen. The temperature was 101 . The bowels were obstinately constipated. July 31 , the general condition was worse; pain had increased and vomiting had occurred. The patient was removed to a hospital for further observation and probable operation. The blood count revealed 23,350 leukocytes. The patient was prepared for operation, which was performed, July 31.

A medial incision was made, and a tumor the size of a large orange, blackened and with a twice-twisted pedicle, involving the left ovary, was removed. The appendix was inflamed and enlarged, and was also removed. The tumor, a dermoid cyst, contained portions of fetus, including hairy scalp, a part of the upper jaw, and six teeth such as those of a 3 year old child. The age of the patient is the chief point of interest. The condition seems to have been reported in only one younger patient, who was $3 \frac{1}{2}$ years of age. The patient next in age was 11 years old.

10924 Prospect Avenue.

\section{New and Nonofficial Remedies}

The FOLLOWING ADDITIONAL ARTICLES HAVE BEEN ACCEPTED as CONFORMing to the rules of the CoUnctl on Pharmacy and Chemistry of the American Medical Association for admission to New and NoNofficial Remedies. A copy of THE RULES ON WHICH THE COUNCIL BASES ITS ACTION WILL BE SENT ON APPLICATION.

W. A. Puckner, Secretary.

CALCIUM CACODYLATE-IPCO (See New and Nonofficial Remedies, 1922, p. 55).

The following dosage forms have been accepted:

Ven Calcium Cacodylate Ampules, $3 / 4$ grain.-1 Cc. contains calcium cacodylate-I pco, $0.05 \mathrm{Gm}$. (3/4 grain).

Ven Calcium Cacodylate Ampules, $1 \frac{1}{2}$ grains. 1 Cc. contains calcium cacodylate-Ipco, $0.097 \mathrm{Gm}$. (11/2 grains).

Ven Calcium Cacodylate Ampules, 3 grains. -1 Ce. contains calcium cacodylate-I pco, $0.195 \mathrm{Gm}$. (3 grains).

Ven Calcium Cacodylate Ampules, 5 grains.-1 Cc. contains calcium cacodylate-Ipco, $0.324 \mathrm{Gm}$. (5 grains).

Ven Calcium Cacodylate Ampules, 7 grains, -1 Cc. contains calcium acodylate-Ipco, $0.453 \mathrm{Gm}$. (7 grains).

MERCURIALIZED SERUM-LEDERLE (See New and Nonofficial Remedies, 1922, p. 189).

The following dosage form has been accepted:

Mercurialized Serum-Lederle for Intrazenous Injection.-Each package contains the equivalent of $1 / 3$ grain $(0.022 \mathrm{Gm}$.) of mercuric chloride in $8 \mathrm{Cc}$. normal horse serum. The initial dose is $1 / 12$ grain $(0.0055 \mathrm{Gm}$.) of the mercuric chloride. This may be gradually increased to $1 / 3$ grain $(0.022 \mathrm{Gm}$.). The injections may be given at intervals of about one week.

PROTARGIN MILD (See New and Nonofficial Remedies, 1922, p. 326).

Silvol.-A brand of protargin mild-N. N. R. Silvol is a compound of colloidal silver with an alkaline proteid and contains about 20 per cent. of silver.

Actions, Uses and Dosage.-See general article, Silver Preparations, New and Nonofficial Remedies, 1922, p. 321.

Manufactured by Parke, Davis and Company, Detroit. No U. S. patent or trademark

Psychoses in Factories and Offices.-Recently repeated instances of mass psychoses have been reported from factories and offices, in Vienna, according to a correspondent, mostly among girls; but, in several instances, men have fallen victims. The first observations were made in a government central telephone station. An operator fainted and had convulsions. Within a few minutes, more than a dozen of the girls were on the floor in convulstons, and the disturbance spread so rapidly that the station had to be closed for a while owing to lack of operators. The imitative coercion was so strong that even some men had convulsions. A similar occurrence took place shortly afterward in another office, the postal savings bank. Here, lessened resistance to psychic infection, due to underfeeding and overwork, was considered responsible for the occurrence, as the operators are none too well paid, being in state employment. But quite recently simil $\mathrm{r}$ incidents took place in an electric factory, where the wages paid are sufficient to insure proper feeding and little worry. The next day, the same thing occurred in another factory, but was soon checked by the energetic action of the foreman, who at once applied hydrotherapeutic measures. Undoubtedly, the high tension in which the population of this town is living, together with frequent abuse of alcohol, distractions and perhaps the restricted moral control of family life, is responsible for the spread of these psychic disturbances. 